\title{
Editorial: Adverse and Toxic Effects of Childhood Cancer Treatments
}

\author{
Antonio Ruggiero ${ }^{1 *}$, Roderick Skinner ${ }^{2,3}$ and Wael Zekri Khaled Zekri ${ }^{4}$ \\ 1 Pediatric Oncology Unit, Fondazione Policlinico Universitario Agostino Gemelli Istituto di Ricovero e Cura a Carattere \\ Scientifico (IRCCS), Università Cattolica del Sacro Cuore, Rome, Italy, ${ }^{2}$ Department of Paediatric and Adolescent \\ Haematology/Oncology, Great North Children's Hospital, Newcastle, United Kingdom, ${ }^{3}$ Newcastle University Centre for \\ Cancer, University of Newcastle, Newcastle, United Kingdom, ${ }^{4}$ National Cancer Institute, Cairo University and Children \\ Cancer Hospital, Cairo, Egypt
}

Keywords: children, adverse effects, cancer, treatment, fertility

Editorial on the Research Topic

Adverse and Toxic Effects of Childhood Cancer Treatments

The development of effective treatments for cancer has been a landmark achievement of medical research in the modern era. Now, many children with cancer can be cured with dramatic increases in the survival rates of most types of childhood cancers over the last 30 years (1). Most antineoplastic drugs can produce acute toxicities (i.e., nausea, vomiting, mucositis, alopecia,...) which are generally reversible once the chemotherapy is completed (2-4). Some anticancer drugs are associated with delayed toxic effects which can became evident even many years from the chemotherapy $(5,6)$. Therefore, as the number of survivors from childhood cancers is increasing, late effects are now becoming major concerns highlighting the importance of long-term follow-up $(7,8)$.

This Research Topic includes 12 manuscripts focusing on the pathophysiology, diagnosis, treatment, and outcomes of acute and long-term side effects in children treated for cancer.

In their retrospective study, $\mathrm{Lu}$ et al. aimed to investigate the relationships between the methylenetetrahydrofolate reductase (MTHFR) C677T/A1298C and high-dose methotrexate (HD-MTX)-related toxicities in a homogenous group of children with non-Hodgkin lymphoma (NHL). Patients harboring mutant C677T genotype were more vulnerable to oral mucositis, leucopenia, and thrombocytopenia while those with mutant A1298C genotype were more likely to develop anemia and leucopenia but less susceptible to vomiting. These results can be of great interest for clinicians as predictors of MTX toxicity can help to determine the appropriate individual dosage minimizing adverse effects.

Attinà et al. reported their experience of the management of oral mucositis as well as documenting its risk factors. Mucositis was a common complication of treatment for childhood malignancies with $50 \%$ of patients suffering from at least one episode. Its occurrence was related to the presence of neutropenia, number of chemotherapy courses, and type of tumor. The WHO oral mucositis scale appeared to be a valuable tool for assessing the severity of mucositis and the relative effectiveness of pain relief with opioids.

The study by Blom $\mathrm{T}$ et al. provides a focus on the treatment-related toxicities during immunotherapy with dinutuximab, IL-2, GM-CSF, and isotretinoin in 26 high-risk neuroblastoma patients receiving treatment according to the DCOG NBL2009 protocol. The most common grade $\geq 3$ toxicities were pain, central venous catheter-related infections, and fever. 
In total, 310 grade $\geq 3$ toxicities were recorded in 124 courses and a higher number of toxicity episodes was observed in children receiving IL-2.

With a broad perspective on its potential for cancer treatment, the review by Cerchione et al. outlines the role of Dasatinib in the management of childhood Philadelphia chromosome-positive acute lymphoblastic leukemia (Ph+ALL). The use of the second generation tyrosine kinase inhibitors (TKIs), such as Dasatinib, has significantly improved the outcome for pediatric patients with $\mathrm{Ph}+\mathrm{ALL}$ when used in combination with the standard chemotherapy protocols. The role of Dasatinib in combinations with other molecules for new risk-adapted/MRD-driven clinical trials in relapsed $\mathrm{Ph}+\mathrm{ALL}$.or in a post hemopoietic stem cell transplantation setting has to be investigated in future studies.

Several articles within this Research Topic explore recent developments in fertility preservation. Brancati et al. underline the variations in access to available preventive measures. Pharmacological treatment with GnRHa has become an alternative non-invasive and well-tolerated approach, especially in those who cannot access cryopreservation options due to clinical and/or logistic issues. Nevertheless, at present, only very limited evidence from randomized clinical trials on the use of GnRHa in adolescents with cancer is available. In their translational research model, Hao et al. explore the feasibility of follicle isolation and culture from prepubertal mice ovaries recently treated with cyclophosphamide as an alternative fertility preservative method. In addition, the reproductive potential of these follicles regarding the final achievement of mature competent oocytes has been also studied. The follicles obtained were able to grow, and had spindle and chromosome configurations that were normal in the mature oocytes. Wikander et al. report their prospective study involving prepubertal and adolescent girls undergoing HSCT and the outcomes of fertility preservation treatments performed before or after HSCT. Although, the study has some limitations due to the small size, authors were able to demonstrate that fertility preservation can be achieved before and also after HSCT so enhancing the chances of future pregnancies.

Camet et al. assessed the effect of dosing, infusion times, and schedules of cisplatin administration on hearing loss in children receiving cisplatin. Their findings show that the amount of cisplatin infused per dose was strongly associated with an increased risk of hearing loss as well as cumulative cisplatin dose and young age at treatment.

Evidence is accumulating that for childhood cancer survivors, late effects including cardiotoxicity, chronic health conditions, neurocognitive and behavioral functioning can have a negative

\section{REFERENCES}

1. Gatta G, Botta L, Rossi S, Aareleid T, Bielska-Lasota M, Clavel J, et al. Childhood Cancer Survival in Europe 1999-2007: Results of EUROCARE-5 -a Population-Based Study. Lancet Oncol (2013) 15:35-47. doi: 10.1016/ S1470-2045(13)70548-5 impact on their quality of life. Blom JM et al. suggest the adoption of digital phenotyping and dynamic monitoring for adolescents treated for cancer. Their integrated multidimensional model, as part of a digital toolbox, can represent a new approach for the development of age-appropriate resources that could help them in managing their disease and the treatment side effects. The work of Sofia et al. aimed at detecting late subclinical cardiac dysfunction in children treated for cancer with anthracyclines. The early recognition of such sub clinical cardiac dysfunction may be fundamental for facilitating prompt appropriate medical management. They did not identify statistically significant correlations between echocardiographic parameters $(2 \mathrm{D}$, strain and $3 \mathrm{D}$ assessment) and age at cancer diagnosis or duration of follow-up. Significantly reduced 3D left ventricle ejection fraction was reported in children treated with anthracyclines despite no significant differences in 2D ejection fraction and longitudinal strain values.

Ewig et al. conducted a retrospective study on chronic polypharmacy prescription among childhood cancer survivors. They confirm the potentially high incidence of late, and often permanent, health complications arising from intensive treatment with combination chemotherapy and ionizing radiation especially for children with central nervous system tumors or survivors who undergone hematopoietic stem cell transplantation. For physicians who are dedicated to taking care of these patients, future work requires appropriate consideration for this vulnerable population.

In their prospective study on Chinese survivors of childhood acute lymphoblastic leukemia (ALL), Peng et al. detected that most young survivors had normal cognitive and behavioral function during the early phase of survivorship although subjects with chronic health conditions or negative socio-environmental were at higher risk of executive cognitive dysfunction.

Although the studies presented in this Research Topic cannot comprehensively cover the whole breadth of this topic, the result of each individual study opens new research and clinical questions helpful to build a new health system model aimed at reducing the risk of major adverse and toxic effects related to cancer treatment.

\section{AUTHOR CONTRIBUTIONS}

AR conceived the draft. RS and WK contributed to the manuscript text and editing. RS supervised the manuscript writing process. All authors provided critical feedback and helped shape the direction of the manuscript. Chemotherapy-Induced Nausea and Vomiting in Children With Cancer: Still Waiting for a Common Consensus on Treatment. J Int Med Res (2018) 46 (6):2149-56. doi: 10.1177/0300060518765324

3. Dunnill CJ, Al-Tameemi W, Collett A, Haslam IS, Georgopoulos NT. A Clinical and Biological Guide for Understanding Chemotherapy-Induced 
Alopecia and Its Prevention. Oncologist (2018) 23(1):84-96. doi: 10.1634/ theoncologist.2017-0263

4. Rinninella E, Ruggiero A, Maurizi P, Triarico S, Cintoni M, Mele MC. Clinical Tools to Assess Nutritional Risk and Malnutrition in Hospitalized Children and Adolescents. Eur Rev Med Pharmacol Sci (2017) 21(11):2690-701.

5. Ehrhardt MJ, Skinner R, Castellino SM. Renal and Hepatic Health After Childhood Cancer. Pediatr Clin North Am (2020) 67(6):1203-17. doi: 10.1016/j.pcl.2020.07.011

6. Timeus F, Crescenzio N, Longoni D, Doria A, Foglia L, Pagliano S, et al. Paroxysmal Nocturnal Hemoglobinuria Clones in Children With Acquired Aplastic Anemia: A Multicentre Study. PloS One (2014) 9(7):e101948. doi: 10.1371/ journal.pone.0101948

7. van Kalsbeek RJ, Mulder RL, Skinner R, Kremer LCM. The Concept of Cancer Survivorship and Models for Long-Term Follow-Up. Front Horm Res (2021) 54:1-15. doi: 10.1159/000514693

8. Shapiro CL. Cancer Survivorship. N Engl J Med (2018) 379(25):2438-50. doi: 10.1056/ NEJMra1712502
Conflict of Interest: The authors declare that the research was conducted in the absence of any commercial or financial relationships that could be construed as a potential conflict of interest.

Publisher's Note: All claims expressed in this article are solely those of the authors and do not necessarily represent those of their affiliated organizations, or those of the publisher, the editors and the reviewers. Any product that may be evaluated in this article, or claim that may be made by its manufacturer, is not guaranteed or endorsed by the publisher.

Copyright $\odot 2021$ Ruggiero, Skinner and Khaled Zekri. This is an open-access article distributed under the terms of the Creative Commons Attribution License (CC BY). The use, distribution or reproduction in other forums is permitted, provided the original author(s) and the copyright owner(s) are credited and that the original publication in this journal is cited, in accordance with accepted academic practice. No use, distribution or reproduction is permitted which does not comply with these terms. 